\title{
Identificación Mediante Huellas Labiales: Casos Paradigmáticos, Oportunidades Perdidas y "Anomalías" para la Construcción de un Nuevo Paradigma
}

\author{
Lip Print Identification: Paradigm Case Argument, Lost Opportunities \\ and "Anomalies" for the Construction of a New Paradigm
}

Gabriel M. Fonseca'; Carlos Ramírez-Lagos²; Jorge Ortiz-Contreras \& Sandra López-Lázaro

FONSECA, G. M.; RAMÍREZ-LAGOS, C.; ORTIZ-CONTRERAS, J. \& LÓPEZ-LÁZARO, S. Identificación mediante huellas labiales: Casos paradigmáticos, oportunidades perdidas y "anomalías" para la construcción de un nuevo paradigma. Int. J. Odontostomat., 12(2):169-176, 2018.

RESUMEN: El análisis de huellas labiales con fines de identificación criminal ha sido denominado Queiloscopía, especialidad de la odontología forense que se ha sustentado en las supuestas unicidad, perennidad y clasificabilidad de los patrones de surcos labiales. En la actualidad, dos elementales corrientes parecen representar la realidad de la queiloscopía en el mundo forense: mientras algunos casos han demarcado su construcción estableciendo un paradigma de gran poder en la segunda mitad del siglo XX, la insuficiencia técnica en algunos casos o la presencia de nuevos estándares jurídicos en otros han puesto en jaque a esta subdisciplina de la Odontología Forense. Se presenta una reevaluación de los paradigmas propuestos por esos casos, se exponen situaciones fallidas en ese tipo de manejo de evidencias y se presenta una proyección efectiva local a esta (aún) potencial herramienta de investigación criminal.

PALABRAS CLAVE: odontología forense, huellas labiales, identificación criminal.

\section{INTRODUCCIÓN}

El análisis de las huellas dejadas por los labios y sus surcos es conocido como Queiloscopía, del griego "cheilos" (labios) y "skopein" (observar) (Dolly et al., 2016). Se ha mencionado que su mayor importancia refiere al carácter único de esas huellas, y a que sus surcos son permanentes y no sufren modificaciones con el tiempo (salvo en condiciones patológicas específicas) (Caldas et al., 2007). Los análisis queiloscópicos han sido fuertemente relacionados con un valor identificatorio similar, según la literatura, al de las huellas dactilares, relación que ha sido abordada conceptual y técnicamente (Negi \& Negi, 2016).

Sólo como planteamiento contextual, la búsqueda del término "lip print" AND "forensic" AND "identication"-, arroja a la fecha (27 de marzo de 2018), la interesante cifra de 85 resultados en Google Aca- démico, y de 9 resultados en PubMed/Medline, esto sólo contando desde el 1 de enero de 2017. Esta pesquisa da cuenta del atractivo que representa esta particular forma de rastro en la investigación criminal; sin embargo ya ha sido mencionado que los análisis queiloscópicos necesitan de una investigación más profunda y de diseños experimentales que permitan no solo desarrollar perfiles antropológicos sino también obtener herramientas que permitan usarlos en situaciones reales de cotejo (Fonseca et al., 2014). Se ha afirmado que, aunque existen suficientes casos reportados de identificación criminal mediante el uso de esta evidencia, se hacen necesarias las revisiones de estos reportes -a la vista de los nuevos paradigmas jurídicos-, y de la puesta a punto de sus técnicas, esta vez aplicadas convenientemente en diseños empíricos que permitan su contrastación (Fonseca et al.).

\footnotetext{
${ }^{1}$ Laboratorio de Pericias en Odontología Forense (LPO), Centro de Investigación en Odontología Legal y Forense (CIO), Facultad de Odontología, Universidad de La Frontera - Temuco, Chile

${ }^{2}$ Laboratorio de Criminalística de Carabineros de Chile (LABOCAR) - Temuco, Chile

Trabajo financiado parcialmente por la Universidad de La Frontera, Proyecto DI16-0112
} 
FONSECA, G. M.; RAMÍREZ-LAGOS, C.; ORTIZ-CONTRERAS, J. \& LÓPEZ-LÁZARO, S. Identificación mediante huellas labiales: Casos paradigmáticos, oportunidades perdidas y "anomalías" para la construcción de un nuevo paradigma. Int. J. Odontostomat., 12(2):169-176, 2018.

En 2014 presentamos una primera revisión de casos con utilización de evidencia queiloscópica (Fonseca et al.); se presenta a continuación una reevaluación de los paradigmas propuestos por esos casos, se exponen situaciones fallidas en ese tipo de manejo de evidencias y se presenta una proyección efectiva local a esta (aún) potencial herramienta de investigación criminal.

\section{Los casos paradigmáticos}

La Real Academia Española define a "paradigma" en su segunda acepción como una "teoría o conjunto de teorías cuyo núcleo central se acepta sin cuestionar y que suministra la base y modelo para resolver problemas y avanzar en el conocimiento". Probablemente sea una elucubración del pionero francés Edmond Locard cuando sugiere identificar las huellas labiales de cartas firmadas con labios pintados o del extremo de cigarrillos (Locard, 2010), la que sembró la inquietud a mediados de los años '40 de aplicar esta experticia en campo. Reconocido como la primera utilización documentada de huellas labiales en un caso forense no sólo en los Estados Unidos sino también a nivel global (Suzuki \& Tsuchihashi, 1970), el caso del atropello y fuga investigado por el Teniente Leland V. Jones (del Laboratorio de Criminalística de la Policía de Los Angeles) en 1945, quizás sea la mejor representación del paradigma queiloscópico. Ese año, una muchacha fue atropellada por un Dodge coupé y seriamente lesionada. La víctima fue arrojada sobre el capot del automóvil rebotando y tomando contacto sus labios con la parte superior del guardabarros delantero derecho. A partir de declaraciones de testigos finalmente fue localizado el automóvil y, luego de ser procesado por los investigadores logró identificarse en ese sector una perfecta huella labial. El rastro fue levantado con cinta adhesiva transparente y perennizado sobre fondo blanco pues el color del guardabarros dificultaba una correcta fotografía. La impresión levantada fue enviada al laboratorio y analizada por Jones. Este comparó el rastro con dos ejemplares obtenidos directamente de los labios de la víctima hospitalizada. Los 17 puntos de coincidencia entre los rastros fueron suficientes para acusar al conductor quien finalmente se declaró culpable del delito (Jones, 1959, 1964).

Jones ganó un gran prestigio entre sus colegas (debe mencionarse que el Laboratorio de Criminalística de la Policía de Los Ángeles fue el primero en su tipo en los Estados Unidos)
(Hertzberg-Davis Forensic Science Center, 2007); aunque para 1954 Jones ya no practicaba su especialidad primaria (el análisis comparativo de impresiones), él había adquirido gran experticia en el reconocimiento de evidencia física y en la valoración probatoria de diversas formas de rastros (Cadman, 1990). Su retiro de la policía de Los Angeles en 1957 luego de 21 años no le impidió continuar como consultor de la institución. Con más de 2.500 presentaciones en tribunales penales, Jones continuó como profesor asociado en algunas universidades estatales californianas y ayudó a consolidar la fundación de la California Association of Criminalists (CAC) (Cadman). El caso en cuestión fue compilado y publicado por el prestigioso médico legista LeMoyne Snyder en 1950 (y en sucesivas reediciones, siempre atribuyendo a Jones el trabajo pericial) (Snyder, 1950), lo que a futuro desdibujaría la real historia y autoría del caso (Fonseca et al.) (Fig. 1).

El segundo caso paradigmático aconteció en octubre de 1979 en San Diego, California. Mientras los clientes de una pequeña sucursal del Bank of America en Bonita Ranch llevaban a cabo sus operaciones, las cámaras de seguridad registraron un particular evento: Una mujer afroamericana, después de robar 10.500 dólares, golpeó con su cara una de las puertas de vidrio del banco al escapar. En la investigación, los detectives examinaron esa puerta corroborando la presencia de una perfecta huella labial. Mediante procedimientos dactiloscópicos, los técnicos utilizaron cinta adhesiva transparente para levantar el rastro y fijarlo en fondo de papel blanco. Una segunda muestra fue revelada con polvo negro y fotografiada, y ambas huellas fueron enviadas a la división de dactiloscopía del Federal Bureau of Investigation (FBI). Si bien un llamado anónimo denunció a Jonathan Harvey Eugene Jackson, de 26 años como responsable del robo (ya tenía antecedentes criminales), los testigos afirmaban haber visto a una mujer y no a un hombre cometer ese crimen. Esto no detuvo a los detectives y Jackson fue detenido. Los técnicos solicitaron a Jackson el colocarse el mismo lápiz labial identificado en la puerta del banco y tomaron huellas, las que fueron confrontadas con las de la de la escena encontrando patrones coincidentes suficientes para inculpar a Jackson. Un año después fue declarado culpable y sentenciado a 10 años en prisión. Los agentes de policía declararon que "esa fue la primera vez que el procedimiento fue utilizado por el FBI para aprehender a un sospechoso" (Chula Vista Star-News, 1980; Fonseca et al.). 


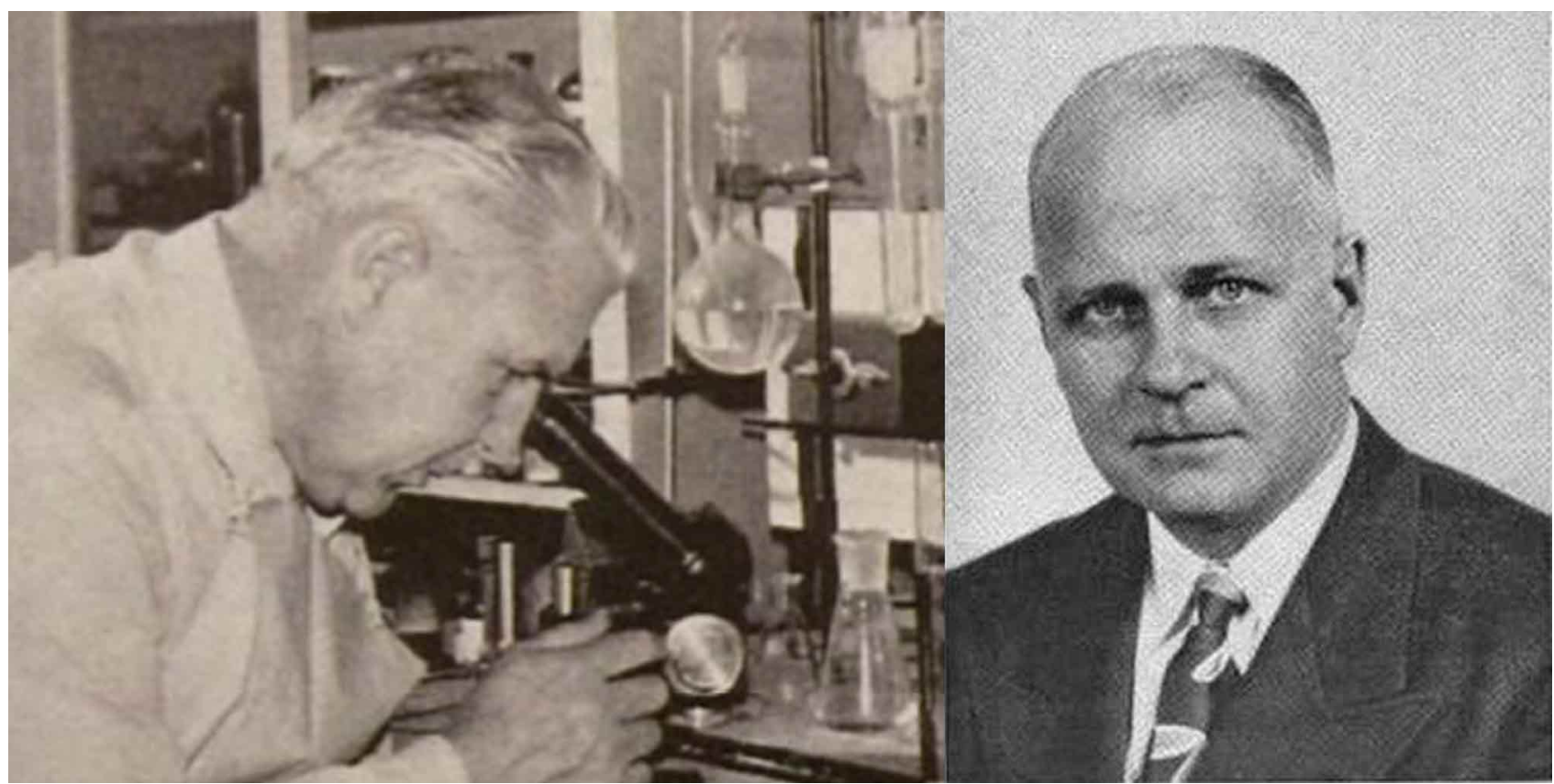

Fig. 1. Izq. Leland V. Jones, del Laboratorio de Criminalística de la Policía de Los Angeles, Estados Unidos (Fotografía: Archivo de la CAC). Der. El Dr. LeMoyne Snyder, de los Laboratorios de Criminalística de la Policía del Estado de Michigan, Estados Unidos (Fotografía: The Michigan Alumnus, 1954).

\section{Las oportunidades perdidas}

Así y todo, no ha sido sencillo (ni tan claro) el camino recorrido por la queiloscopía en la comunidad científica. En 2009, la National Academy of Sciences de los Estados Unidos (NAS) admitía que las huellas labiales eran un tipo de rastro muy poco frecuente, y por esa razón sumamente difícil de ser validadas como evidencia, o al menos para afirmar suficiencia en su variabilidad (Committee on Identifying the Needs of the Forensic Science Community, 2009). Sin embargo, en 2014 cuestionamos esa afirmación por circunstancial y poco objetiva: ¿hasta qué punto puede hablarse de "infrecuencia" si los laboratorios de criminalística o los equipos policiales para la investigación de rastros podían no estar entrenados para reconocer en la escena o para procesa este tipo de evidencia? (Fonseca et al.). Según nuestra propia experiencia, y la de diferentes actores técnicos y de laboratorio de fuerzas policiales en Argentina y Chile, sobran las "anécdotas" de huellas labiales halladas en escenas criminales que, aún identificadas como "potenciales evidencias", no fueron procesadas -o incluso desestimadas- por no contar con los adecuados conocimientos técnicos o empíricos para tal efecto. Estas "oportunidades perdidas" también han sido reportadas en la literatura.

W. J. Pertson, Sargento de la Real Policía Montada Canadiense, relata en 1984 que su interés en las huellas labiales comenzó en 1984, cuando fue convocado para procesar la escena de una casa forzada. "El examen de una ventana a la izquierda de la puerta trasera reveló la impresión parcial de un labio humano así como también la impresión parcial de una oreja derecha arriba y justo a la derecha de la impresión labial. En el medio del área enmarcada por estas huellas había una pequeña mancha que luego se identificó como hecha por la punta de una nariz". Según Pertson, esas huellas las había dejado un individuo con su cara en la ventana mientras espiaba el interior de la casa antes de ingresar forzando la puerta trasera. Aunque procesó las huellas con reveladores de huellas dactilares, desestimó el hallazgo por no considerarlo útil. Sin embargo, el autor reconoce que ese caso despertó su curiosidad, la que lo llevaría a más adelante a considerar las huellas labiales, de las orejas y de la nariz, "no sólo útiles para la identificación criminal, sino también para la identificación positiva de víctimas de desastres, de muertes súbitas o de homicidios" (Pertson, 1987).

Algo similar relata Lincoln C. Petersen en 2009: "Mientras realizaba tareas como novato investigador de la División de Investigación Criminal (de la Marina de los Estados Unidos), una de las primeras 
FONSECA, G. M.; RAMÍREZ-LAGOS, C.; ORTIZ-CONTRERAS, J. \& LÓPEZ-LÁZARO, S. Identificación mediante huellas labiales: Casos paradigmáticos, oportunidades perdidas y "anomalías" para la construcción de un nuevo paradigma. Int. J. Odontostomat., 12(2):169-176, 2018.

escenas de crímenes a las que respondí fue la de un caso de voyeurismo. El experimentado investigador con el que estaba me ordenó que buscara en la escena criminal cualquier posible elemento de valor probatorio. (...) Mientras continuaba mi examen de la ventana usada por el perpetrador para mirar dentro, noté, en el lado exterior del cristal de la ventana, una marca inusual. Tras un examen más detenido descubrí que su forma era similar a la de un conjunto completo de labios. Después de informar al investigador principal y más experimentado que había identificado una posible huella latente labial en la ventana, él me dijo que lo descartara, porque incluso si la marca era una impresión labial, no había sistema conocido para compararla e identificar a un sospechoso". Petersen agrega: "Pensé que sería beneficioso, al menos, levantar el rastro en caso de que un sospechoso fuera posteriormente aprehendido para poder cotejarlas con sus labios; sin embargo, y debido a que yo era nuevo en el campo y sin suficiente experiencia, preferí mantenerme en silencio. Efectivamente, unas semanas más tarde, un sospechoso fue identificado en otra escena criminal similar. Debido a que él prefirió mantenerse en silencio, y al no haber evidencia sustancial que lo vinculara a ninguno de los crímenes, no fue procesado como sujeto" (Petersen, 2009).
Jerzy Kasprzak, de la Escuela de Policía Militar en Min'sk Mazowiecki en Polonia, comenta que en ese país, el interés en las huellas labiales comenzó en 1966 cuando, accidentalmente, una huella labial fue revelada en la ventana de vidrio de la escena de un robo. Refiere Kasprzak: "El experto que realizó el examen no tenía experiencia alguna en la identificación mediante huellas labiales ya que la información sobre la investigación japonesa (llevada a cabo por los precursores Suzuki \& Tshuchihashi) Ilegaría a Polonia a principios de los años setenta. Como resultado del análisis llevado a cabo en 1966, el perito concluyó que el rastro labial revelado en la escena no pertenecía al sospechoso". Sin embargo, Kasprzak agrega: "Hoy, con el material conservado desde ese momento y con los nuevos métodos de pericia queiloscópica, podría afirmarse inequívocamente que el rastro sí pertenecía a ese sospechoso" (Kasprzak, 1990) (Fig. 2).

\section{DISCUSIÓN}

Aquí sería interesante retornar a la Real Academia Española cuando define a "oportunidad" como el "momento o circunstancia oportunos o convenien-

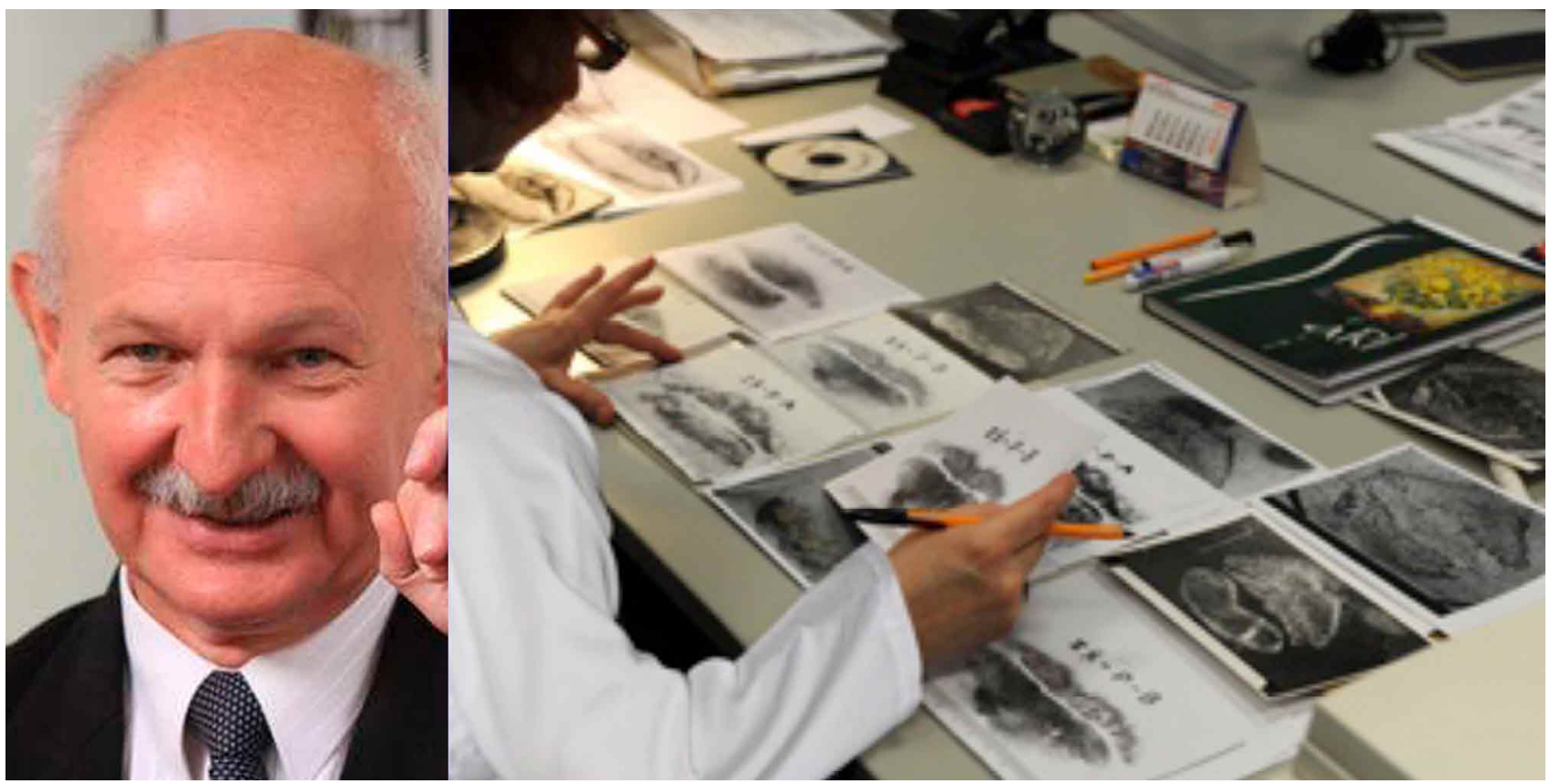

Fig. 2. Izq. El Prof. Jerzy Kasprzak, del Laboratorio Central Forense de la Policía en Varsovia, Polonia (Fotografía: Gazeta Olsztynska, edición del 14 de septiembre de 2013 disponible en http://gazetaolsztynska.pl/171612,Potrafi-znalezc-zabojcepo-sladach-ust-i-uszu.html - axzz5B96CfKZg). Der. Trabajo queiloscópico llevado a cabo por la división especial del Instituto de Investigación del Equipo de Identificación Dactiloscópica del Laboratorio Forense de la Policía Central, Polonia (Fotografía: Instituto de Investigación del Laboratorio Forense de la Policía Central, disponible en http://clk.policja.pl/clk/clkp/ struktura/zaklad-daktyloskopii/65738,Zespol-Identyfikacji-Daktyloskopijnej.html). 
FONSECA, G. M.; RAMÍREZ-LAGOS, C.; ORTIZ-CONTRERAS, J. \& LÓPEZ-LÁZARO, S. Identificación mediante huellas labiales: Casos paradigmáticos, oportunidades perdidas y "anomalías" para la construcción de un nuevo paradigma. Int. J. Odontostomat., 12(2):169-176, 2018.

tes para algo". Pertson, Petersen y Kasprzak inician todos sus relatos desde la "curiosidad" o el "interés" despertado por un tipo de rastro para el que no había información y, a partir de esto, la búsqueda de su solución. Kasprzak, nuevamente atinado, menciona que entre los años 1966 y 1985 solo dos pericias queiloscópicas fueron realizadas en Polonia, "aunque las huellas labiales eran encontradas frecuentemente en las escenas criminales" (Kasprzak, 1990). Esto llevó a que el Departamento de Huellas Dactilares del Laboratorio Central Forense de la Policía en Varsovia buscara protocolizar los análisis queiloscópicos con la puesta a punto en condiciones de laboratorio que permitiera su empleo en los tribunales. Entre 1986 y 1987, dos pericias queiloscópicas fueron realizadas con resultados positivos y categóricos. En 1988, hasta mediados de abril, dos nuevas pericias fueron realizadas, ambas también con resultados positivos y categóricos (Kasprzak, 1990). Para 1997, el laboratorio ha peritado en 85 casos de huellas labiales, 34 de los cuales fueron de resultados positivos (Kasprzak, 2000). En la actualidad, una división especial del Instituto de Investigación del Equipo de Identificación Dactiloscópica del Laboratorio Forense de la Policía Central de Varsovia, realiza periódicamente pericias queiloscópicas incluso por solicitud de otras policías, participa sistemáticamente en pruebas de competencia en estos procesados, además de supervisar las postulaciones de nuevos candidatos a peritos en la disciplina (http://clk.policja.pl/clk/clkp/struktura/zakladdaktyloskopii/65738, Zespol-IdentyfikacjiDaktyloskopijnej.html). Podría mencionarse además que, el legado del investigador polaco ha sido actualmente asumido por un joven doctorando de la Universidad de Silesia, Lukasz Smacki, quien sobre la base de datos alfanumérica propuesta por Kasprzak (actualmente en uso en la policía de Varsovia), lleva desarrollando un algoritmo para evitar el uso de dispositivos ópticos y poder digitalizar esa base de datos. Dado que el protocolo utilizado por la policía polaca incluye el registro de, al menos, 10 muestras indubitadas, el trabajo de Smacki facilitaría considerablemente los cotejos queiloscópicos (Smacki et al., 2016).

Resulta abrumadoramente peculiar que, aunque se pregone la investigación completa y minuciosa de la escena criminal (Palmer \& Polwarth, 2011), sea todavía la "curiosidad" la que motive decidir tomar en cuenta una huella labial detectada de forma accidental, como potencial evidencia. Los casos están a la vista, las huellas labiales son hallazgo frecuente en las escenas criminales (Kasprzak, 1990) y circunstancias de necesaria investigación dan cuenta del pro- fundo interés de la comunidad científica y de la misma sociedad al respecto. El 16 de marzo de 2005, Inge Lotz, una joven estudiante de matemáticas fue brutalmente asesinada en Stellenbosch (Sudáfrica). No había signos de entrada forzada en su apartamento, ni evidencias de robo o violación. El novio de la víctima, Fred van der Vyver, fue acusado del homicidio. Las pruebas en su contra fueron un martillo hallado en su vehículo (compatible con las lesiones descritas en la víctima), y una huella dactilar dejada en la cubierta de un DVD arrendado la noche anterior al homicidio y dejado en el sitio del suceso. Después de un juicio de 10 meses, y luego de inconsistencias en los testimoniosde los peritos, el acusado fue dejado en libertad. La contrapericia de la defensa instaló la sospecha de manipulación de la prueba. Mientras la policía afirmaba que la huella dactilar había sido levantada de una superficie plana (la cubierta del DVD), la defensa argumentó que en realidad había sido levantada de una superficie curva, probablemente un vaso de vidrio. Eso dejaría fuera de la escena al acusado. En la misma cinta de perennización de las huellas dactilares, se encontró posteriormente una mancha que, según la defensa, sería una huella labial. Esto reforzaría la hipótesis de que el acusado no estuvo en la escena. A todo esto, se sumó que la policía -increíblemente- había devuelto la cubierta del DVD al local de arriendo, con lo que las huellas no pudieron ser verificadas por la contraparte. Tres peritos apoyaron la hipótesis de que esa huella no era una huella labial; nuestro ya conocido L. C. Petersen afirmó: "Después de ver las imágenes no encuentro que esa marca sugiera haber sido producida por un labio". N. H. Hamzah, de Malasia: "Puedo asegurar concluyentemente que la marca no sugiere una huella labial". B. Rai, de la India: "No encuentro ningún patrón asociable a una huella labial". El caso aún sigue severamente cuestionado por los familiares de la víctima (Truth 4 Inge, 2018) (Fig. 3).

Con el número de código DI16-0112, en 2016, nuestro Laboratorio de Pericias en Odontología Forense perteneciente al Centro de Investigación en Odontología Legal y Forense $(\mathrm{ClO})$ de la Facultad de Odontología de la Universidad de La Frontera, inició el proyecto titulado "Evaluación del proceso de revelado, fotografiado y cotejo de huellas labiales latentes en soportes no porosos utilizando cianocrilato Lumicyano $^{\mathrm{TM}}$ para identificación forense". Con una duración de 2 años y resultados en vías de publicación, este proyecto posee como objetivos específicos i) Desarrollar un protocolo específico para el revelado de huellas labiales latentes en soportes no porosos mediante cianocrilato Lumicyano ${ }^{\mathrm{TM}}$; ii) Comparar cali- 


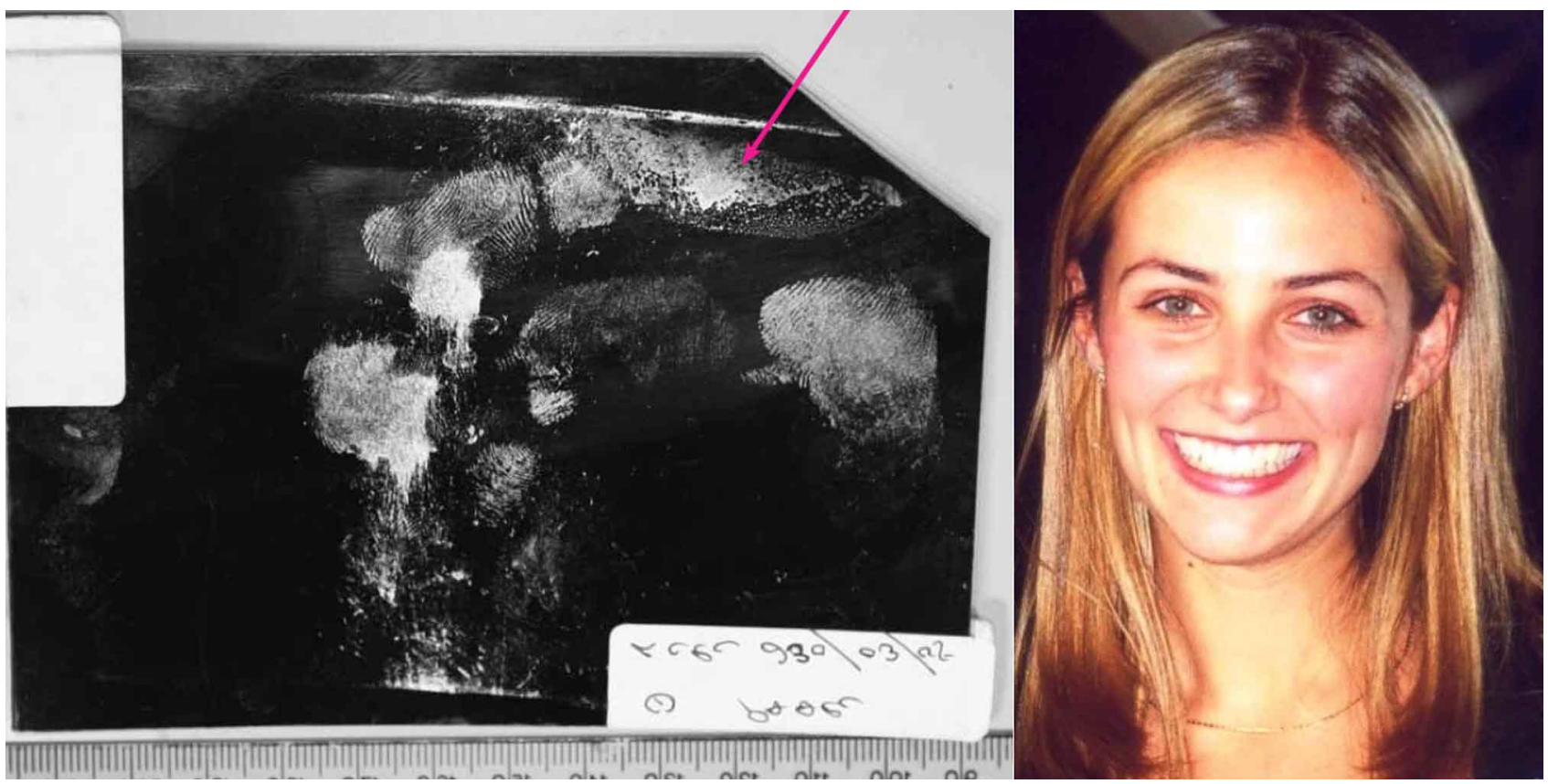

Fig. 3. Izq. Rastros papiloscópicos levantados por la policía en la escena del homicidio de Inge Lotz en Stellenbosch, Sudáfrica. La flecha indica la mancha interpretada por la defensa como una huella labial, la que dejaría fuera de escena al sospechoso Fred van der Vyver Der. Inge Lotz, la joven estudiante asesinada (Fotografías: http://www.justice4inge.com)

dad de revelado de Lumicyano ${ }^{\mathrm{TM}}$ (revelador químico de una fase) con cianocrilato+polvos fluorescentes (revelador químico-físico de dos fases), y con polvos fluorescentes (revelador físico de una fase) para el revelado de huellas labiales latentes; y iii) Estimar valor identificatorio (índice de error conocido o potencial y normas de control) de las huellas relevadas y fotografiadas mediante Lumicyano ${ }^{\mathrm{TM}}$. Más allá de que este proyecto representa un hito en la vinculación de un laboratorio civil (LPO) y uno policial (Laboratorio de Criminalística de Carabineros de Chile -LABOCARTemuco) para investigación forense (Fig. 4), esto representa precisamente una oportunidad de resolver, al menos en parte, algunos de los duros cuestionamientos que la queiloscopía ha recibido a expensas del nuevo paradigma crítico de las ciencias forenses identificatorias: necesidad de contrastación y validación de sus metodologías (Committee on Identifying the Needs of the Forensic Science Community et al.; Moenssens, 2011).

La necesidad de "anomalías" para la construcción de un nuevo paradigma. Las particularidades de un paradigma se encuentran definidas por el cimiento de una teoría, la aparición de "anomalías" que la cuestionan, y la construcción de nuevas decisiones" (Harker, 2015). En 1997, Lavelle L. Davis era condenado por homicidio y sentenciado a 45 años de prisión en Illinois. La única evidencia que lo vinculaba con el crimen era una huella labial encontrada en un rollo de cinta adhesiva cerca del sitio del suceso (People v. Davis, 1999). El primer juicio aceptó la evidencia provista por dos expertos de la policía estatal (una dactiloscopista y una documentólogo, quienes encontraron 13 puntos de coincidencia entre los rastros), los que sustentaron sus pericias declarando que "el FBI y la Policía del Estado de Illinois consideran a las huellas labiales como método de identificación positiva, ya que la técnica ha existido desde los años 1950, que se han escrito artículos sobre el tema, y que no saben de desacuerdos en la comunidad científica forense respecto de su metodología o de su valor identificatorio" (People v. Davis, 1999). Las declaraciones de Leanne Gray y Steven McKasson, los expertos, en clara alusión a los casos de las huellas en el guardabarros y en la puerta del banco, exponían como cimentados 50 años de esa doctrina queiloscópica con fundamentos locardianos ampliamente difundidos en el medio técnico forense de segunda mitad de siglo (el mismo Leland V. Jones acercaba su discurso a la obra de Locard cuando se refería a la identificación por huellas labiales). Sin embargo, la historia fue radicalmente diferente: la declaración experta de dos peritos dactiloscopistas, Andre Moenssens y Michael Sinke, dejó entrever que la identificación por huellas labia- 

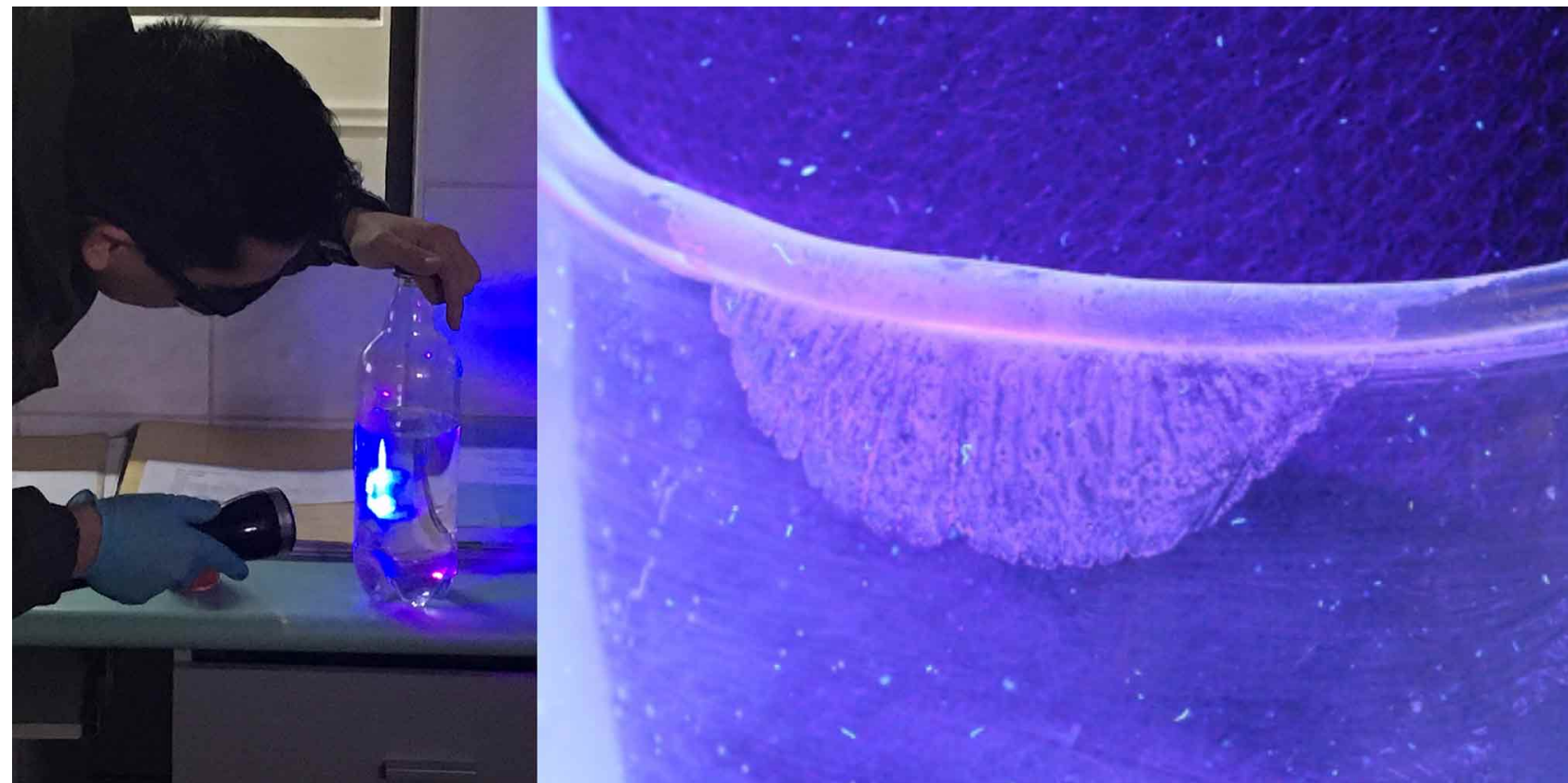

Fig. 4. Izq. Pruebas en condiciones de laboratorio para identificación y revelado de huellas labiales en diferentes soportes (LPO y LABOCAR Temuco). Der. Huella labial en vaso de vidrio, revelada con Lumicyano ${ }^{\mathrm{TM}}$ en cámara de fumigación, expuesta para su fotografía con fuente de iluminación UV (LPO) (Fotografías proyecto DI16-0112).

les no poseía estudios científicos que la validaran ni metodologías aceptadas y normatizadas en la comunidad científica forense. Las declaraciones de Gray y McKasson fueron severamente cuestionadas, y finalmente el 20 de noviembre de 2007, Lavelle L. Davis fue liberado (People v. Davis, 2007). La "anomalía" destrozando el paradigma; pero también una oportunidad de revisión para darle nueva confiabilidad.

"Ciencia Forense" implica dos conceptos estructurales y una tensión implícita entre ellos. Por un lado el concepto de "Ciencia", como apuesta a la evolución con un cuerpo en perpetuo crecimiento donde factores tales como "política", "economía", "guerra", "prensa", "opinión pública", "entretenimiento", incluso "hallazgos casuales", contribuyen dinámicamente a la aparición de "anomalías": puntos críticos que destruyen la confianza en el paradigma prevaleciente y favorecen el desarrollo de otro que logra resolverlos) (Harker). Nadie en su sano juicio dudaría de que mucha de la ciencia considerada válida hace 100 años, hoy sería considerada "basura". Por el otro lado el concepto de "Forense", que traslada todo una transversalidad forzada: de ninguna manera podría condenarse retrospectivamente a alguien por una nueva ley o a la espera de una futura nueva prueba científica. El "hoy" es decisivo y los tribunales están obli- gados a garantizar que la evidencia presentada cumpla con los estándares más actuales y científicos posible para ese momento histórico. Es responsabilidad de los investigadores el amalgamar estos dos conceptos estructurales según esos estándares.

FONSECA, G. M.; RAMÍREZ-LAGOS, C.; ORTIZCONTRERAS, J. \& LÓPEZ-LÁZARO, S. Lip print identification: paradigm case argument, lost opportunities and "anomalies" for the construction of a new paradigm. Int. J. Odontostomat., 12(2):169-176, 2018.

ABSTRACT. Lip print analysis for criminal identification has been called Cheiloscopy, a specialty of forensic odontology based on the alleged uniqueness, durability and classification of labial groove patterns. Presently, there are two basic claims that seem to make reference to Cheiloscopy in the forensic world: While some cases have distinctly marked its design by establishing a dominant paradigm during the second half of the 20th century, others have questioned this particular specialty of Forensic Odontology in view of technical shortcomings or new legal standards. In light of failed cases under this form of evidence management, a reevaluation of the paradigms, and a better use of this potentially effective mechanism in criminal investigation are proposed.

KEY WORDS: forensic odontology, lip prints, criminal identification. 


\section{REFERENCIAS BIBLIOGRÁFICAS}

Cadman, W. J. The formation of the California Association of Criminalists: Genesis of professionalisation? J. F. S. S., 30(3):143-59, 1990.

Caldas, I. M.; Magalhães, T. \& Afonso, A. Establishing identity using cheiloscopy and palatoscopy. Forensic Sci. Int., 165(1):1-9, 2007.

Chula Vista Star-News. Lipstick bandit admits guilt. Chula Vista, Chula Vista Star-News (14 de Septiembre de 1980), 1980. pp.5.

Committee on Identifying the Needs of the Forensic Science Community; Committee on Science, Technology \& Law Policy and GlobalAffairs, Committee on Applied and Theoretical Statistics, Division on Engineering and Physical Sciences. National Research Council of the National Academies. Strengthening Forensic Science in the United States: A Path Forward. Washington DC, The National Academies Press, 2009.

Dolly, A.; Rodrigues, C.; Bankur, R.; Gopinathan, P. A.; Sharma, R. \& Doddamani, A. Evaluation of efficacy of three different materials used in cheiloscopy -A comparative study. J. Clin. Diagn. Res., 10(10):ZC67-71, 2016.

Fonseca, G. M.; Cantín, M. \& Lucena, J. Forensic Dentistry III: Palatal Rugae and Lip Prints in Forensic Identification. Int. J. Odontostomat., 8(1):29-40, 2014.

Harker, D. Creating Scientific Controversies. Uncertainty and Bias in Science and Society. Cambridge, Cambridge University Press, 2015.

Hertzberg-Davis Forensic Science Center. History of the Los Angeles Police Department, Scientific Investigation Division. Los Angeles, Hertzberg-Davis Forensic Science Center, 2007. Disponible en: http://lapdonline.org/history_of_the_lapd

Jones, L. V. Locating and Preserving Evidence in Criminal Cases. In: American Jurisprudence Trials: An Encyclopedic Guide to the Modern Practices, Techniques, and Tactics Used in Preparing and trying Cases, with Model Programs for the Handling of All Types of Litigations, Volume 1. West Group, Bancroft-Whitney Company, Lawyers Cooperative Publishing, Lawyers Cooperative Publishing Company, 1964. pp.563.

Jones, L. V. Scientific Investigation and Physical Evidence. A Handbook for Investigators. Springfield, Charles C. Thomas, 1959.

Kasprzak, J. Cheiloscopy. En: Siegel, J.; Knupfer, G. \& Saukko, P. (Eds.). Encyclopedia of Forensic Sciences. Three-Volume Set, 1-3. East Lansing, Academic Press, 2000.

Kasprzak, J. Possibilities of cheiloscopy. Forensic Sci. Int., 46(12):145-51, 1990.

Locard, E. Manual de Técnica Policíaca. Valladolid, Maxtor, 2010.

Moenssens, A. Lip prints: Admissibility of comparison results. Wiley Encyclopedia of Forensic Science, 2011. Disponible en: http:// onlinelibrary.wiley.com/doi/10.1002/9780470061589.fsa1002/full 2011.

Negi, A. \& Negi, A. The connecting link! Lip prints and fingerprints. J. Forensic Dent. Sci., 8(3):177, 2016.

Palmer, R. \& Polwarth, G. The persistence of fibres on skin in an outdoor deposition crime scene scenario. Sci. Justice, 51(4):1879, 2011.

People v. Davis, 710 N.E.2d 1251 (III.App. 2 Dist. 1999). Leagle Inc., 2017. Disponible en: https://www.leagle.com/decision/ 19991961710ne2d125111874

People v. Davis, 879 N.E.2d 996 (III.App. 2 Dist. 2007). Court Listener, 2017. Disponible en: https://www.courtlistener.com/opinion/ 2050816/people-v-davis/

Pertson, W. J. Ear, lip and nose prints: another means of identification. R.C.M.P. Gazette, 49(9):12-5, 1987.

Petersen, L. C. Lip prints. A thesis submitted to the National
University, La Jolla, CA, USA, March 2006. Anil Aggrawal's Internet J. Forensic Med. Toxicol., 10(1), 2009. Disponible en:http/ /www.anilaggrawal.com/ij/vol_010_no_001/others/thesis/2/ petersen .doc 2009.

Smacki, L.; Luczak, J. \& Wrobel, Z. Lip Print Pattern Extraction Using Top-Hat Transform. In: Burduk, R.; Jackowski, K.; Kurzyn'ski, M.; Wozniak, M. \& Zonierek, A. (Eds.). Proceedings of the 9th International Conference on Computer Recognition Systems CORES 2015. Advances in Intelligent Systems and Computing, vol 403. Cham, Springer, 2016.

Snyder, L. Homicide Investigation: Practical Information for Coroners, Police Officers, and Other Investigators. Springfield, Charles C. Thomas, 1950.

Suzuki, K. \& Tsuchihashi, Y. New attempt of personal identification by means of lip print. J. Indian Dent. Assoc., 42(1):8-9, 1970.

Truth 4 Inge. The "Lip print". Truth 4 Inge, 2018. Disponible en: http:/ /www.justice4inge.com/Lip_report_web_low.pdf

Dirección para correspondencia

Gabriel Fonseca

Laboratorio de Pericias en Odontología Forense (LPO)

Centro de Investigación en Odontología Legal

y Forense $(\mathrm{ClO})$

Facultad de Odontología

Universidad de La Frontera

Temuco

CHILE

Email:gabriel.fonseca@ufrontera.cl

Recibido : 27-03-2018

Aceptado: 30-04-2018 